\title{
Simulation model of the heating and air conditioning system of dc electric trains
}

\author{
Stanislav Istomin ${ }^{1 *}$, and Aleksandr Shtraukhman ${ }^{1}$ \\ ${ }^{1}$ Omsk state transport university, 644046, 35, Marx Ave., Omsk, Russian Federation
}

\begin{abstract}
Most of the territory of the Russian Federation is located in the zone of long-term exposure to negative ambient temperatures. In this regard, in the suburban traffic on the railways of the Russian Federation, a significant proportion of the electric power falls on the operation of heating and air conditioning systems. Nowadays, Russia and the world are developing energy-saving methods and tools to reduce the energy consumption of auxiliary needs of electric trains. In this paper, the method of constructing simulation models in the MATLAB Simulink software was used to build an energy-saving heating and air conditioning system, since this method allows studying various options for building the studied systems with lower financial and labor costs in comparison with the experimental method. The correct selection and display of the parameters of the electric train interior will allow achieving the optimal values of energy consumption for heating and air conditioning of the electric trains. In order to verify its adequacy, the simulation model includes standard values of electric energy consumption for heating and conditioning electric trains for various sections and operating conditions, which were obtained earlier during the correlation and regression analysis of data from electric train parameter recorders. The results of the study showed the adequacy of the application of the developed simulation model for organizing the control of electric power consumption for heating and air conditioning of DC electric trains.
\end{abstract}

\section{Introduction}

Rail transport is a key industry in the Russian Federation, which accounts for about $4.4 \%$ of all electricity generated in Russia, $85 \%$ of which is spent on traction of trains, which is why the priority of the energy strategy of the Russian Railways holding for the period until 2020 and for the prospect until 2030 is a significant increase in the energy efficiency of train traction [1 - 3].

The Russian Federation is one of the largest consumers of energy resources on the Globe (5th place in the world). Its share, according to the results of 2018, accounts for about 889 Terawatt-hours or $4.2 \%$ of all electricity consumed in the world. In this regard, one of the priority areas for the development of science, technology and engineering of the Russian Federation for the next 10-15 years is energy efficiency, energy conservation, and

*Corresponding author: istomin sg@mail.ru 
nuclear energy [4]. It should be noted that the main volume of transportation in electric traction on Russian railways falls on freight traffic and reaches $95 \%$ of the total work. At the same time, the energy consumption for performing such a volume of work is $89 \%$ of the total energy consumption. However, this fact does not mean that the issue of energy conservation in suburban traffic is irrelevant. Given the fact that the activities of subsidiaries of Russian Railways involved in the carriage of passengers in suburban traffic are either unprofitable or are at the break-even point, and the payment of energy resources is the primary expense item for suburban companies, the issue of rational use of energy resources in suburban traffic is very relevant.

In recent years, Russian and foreign scientists have developed a number of methods and tools that allow monitoring and controlling the energy consumption of heating and air conditioning systems:

1. The heating system of the cabin of a direct current electric locomotive [5], comprising a high-voltage electric heater consisting of series-connected tubular heating elements, motor fans, which supply heated air to the workplaces in the cabin, an electric heater control unit and motor fans. The main features of this system are:

- a high-voltage electric heater is placed in the back of an electric locomotive in a tank with a liquid coolant, the motor-fans in the cabin are installed in heaters together with radiators connected to the tank by discharge and supply pipelines, a non-freezing liquid is used as a coolant;

- at least three parallel heaters are installed in the cabin, two of which are installed in the lower part of the rear wall of the cabin opposite the workplaces of the personnel, and one is under the remote control panel and is equipped with air ducts directed to the steps and windows;

- the discharge pipeline has a slope from the tank to the radiators of the heaters, and the supply pipe has the reverse slope, and the reductions in the slopes do not exceed the diameter of the pipe along the entire length of each pipeline.

The main disadvantage of this system is the location of the high-voltage electric heater in the body of an electric locomotive in a tank with a liquid coolant, which can lead to increased heat losses in the pipeline and, as a result, an excessive consumption of electric power.

2. The heating, ventilation and air conditioning system of the driver's cabin of the rail vehicle [6], comprising a heating, ventilation and air conditioning device connected to the distribution and recirculation chambers installed in the roof cave-in area, in which the distribution chamber is connected through the vertical air ducts for the prepared air to the air duct of the heated air, which in turn are connected to a heated air distributor located in front of the cabin below the frontal window.

The main disadvantage of this system is the impossibility of installing an air conditioning system on most series of direct current electric trains due to limited space.

3. A climate control system in the locomotive control cabin [7], comprising an air conditioner connected through power contacts of the contactor to a three-phase highvoltage source of locomotive AC voltage, heaters, which are heating elements and fans with drive motors, electric furnaces, flat heating elements, heating elements which are connected to a three-phase high-voltage source of locomotive AC voltage through circuit breakers and power circuits contactor, IR-aerofit.

4. The device (panel) for controlling the heating of the passenger compartment of a vehicle [8], comprising a control unit connected to temperature sensors, heaters and damper actuators of the ventilation and heating device connected to the air supply channels to the passenger compartment. This device can be used as a prototype in the development of visual control of the temperature in the passenger compartments of electric trains.

5. Temperature sensor for heating systems of the passenger compartments of 
rolling stock with a characteristic of the "hysteresis" type, made in the form of an electrical circuit containing the first and second terminals that form its power input, the third terminal that forms its control output, and a heat-sensitive unit [9]. This sensor can be used as a prototype when developing options for placing sensors in the passenger compartments of electric trains.

6. A dedicated outdoor air system (DOAS) with thermoelectric radiating panels (TERPs) for high-speed train cabins, which prevents air quality problems and reduces energy consumption during operation. The results showed that the proposed system allows saving $32.6 \%$ of the annual working energy [10].

However, each of the above developments requires refinement to adapt to actual specific operating conditions. A preliminary conclusion on the feasibility of implementing certain devices on an electric train can be given by an adequate simulation model of the operation of heating and air conditioning systems, using which it is possible to simulate various options for upgrading systems and assess their effectiveness in terms of energy efficiency.

\section{Object and methods of research}

The object of the study is the heating and air conditioning systems of ED4M electric trains of direct current.

In this paper, the authors used the method of constructing simulation models in the MATLAB Simulink software to build an energy-saving heating and air conditioning system, since this method allows studying various options for building the studied systems with lower financial and labor costs in comparison with the experimental method. The correct selection and display of the parameters of the electric train interior will allow achieving the optimal values of the energy consumption for heating and air conditioning of the electric trains.

The model of the car of the ED4M electric train with a heating and air conditioning system has the following characteristic features:

1) all car systems are excluded, except for the heating system;

2) the electric circuit of the heating system power supply has been replaced with a logical one (1 - power is on, 0 - off)

3 ) in order to verify its adequacy, the simulation model includes the standard values of electric energy consumption for heating and conditioning electric trains for various sections and operating conditions, which were obtained by the authors earlier during the correlation and regression analysis of data from electric train parameter recorders.

\section{Results}

The simulation model of the heating system for the section of the ED4M electric train was built in the MathLAB software environment using SimScape tools related to thermal physical processes. Figure 1 shows a general diagram of a model of a heating and air conditioning system for an electric train section. 

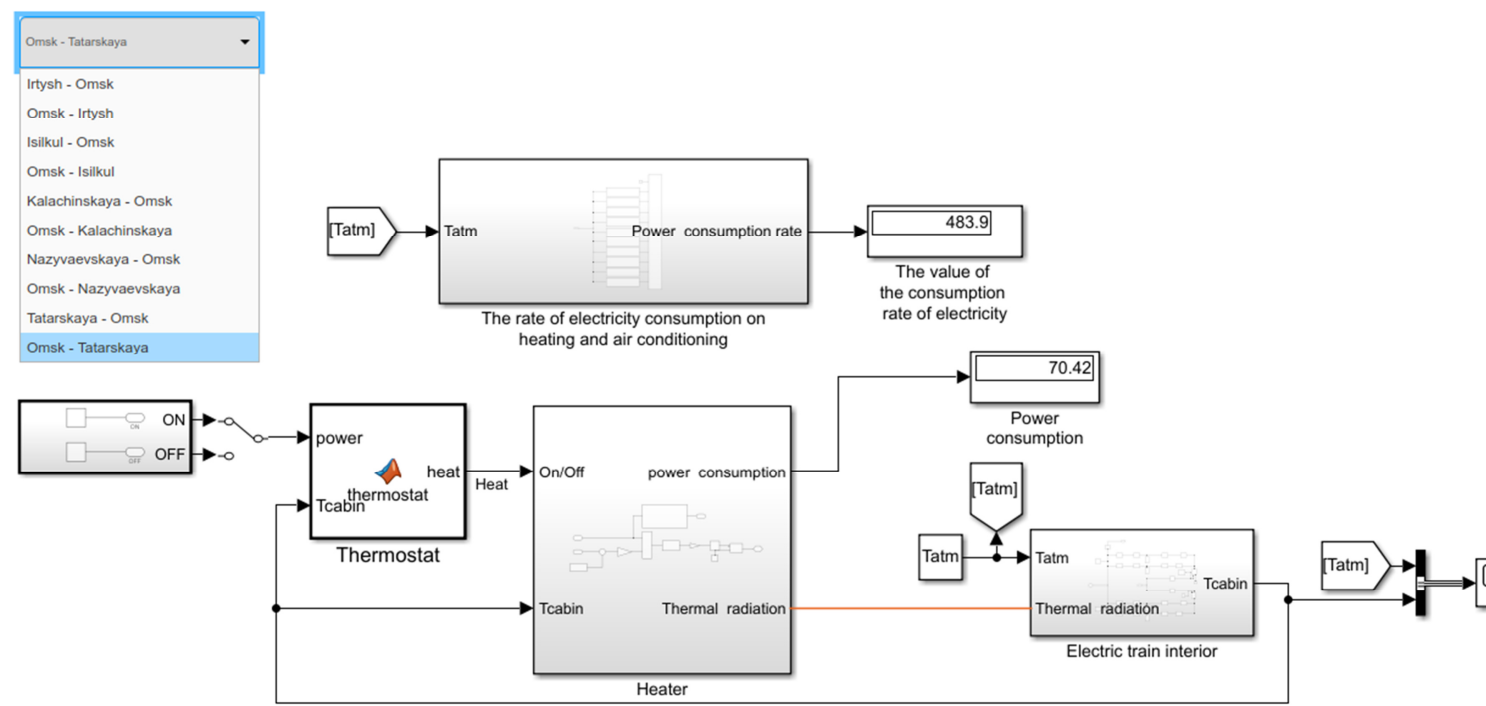

Fig.1. The model of the heating system of the ED4M electric train section

This model consists of the following elements:

1) Battery;

2) Thermostat;

3) Heater (electric heater) and electricity counter;

4) Electricity rating module;

5) Electric train interior.

The battery in this model is presented in the form of logical 0 and 1 . "ON" is 1 , and 0 is "OFF", respectively.

The program of the thermostat operation is shown in Figure 2.

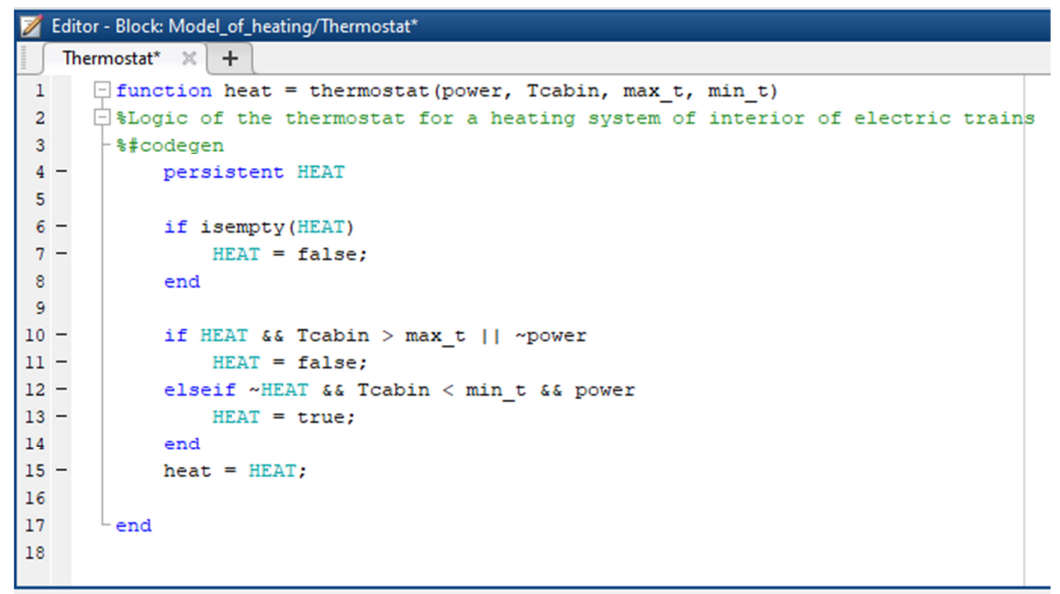

Fig.2. The program of the thermostat operation

The essence of this thermostat lies in the fact that in this unit, there is a comparison of the cabin temperature with the temperature set in the thermostat (max_t and min $t$ with t_cabin). And further, depending on the fulfillment of the condition, a control signal is supplied to the heating element (to the electric heater).

The model of the electric heater and the electricity counter is illustrated in Figure 3. 


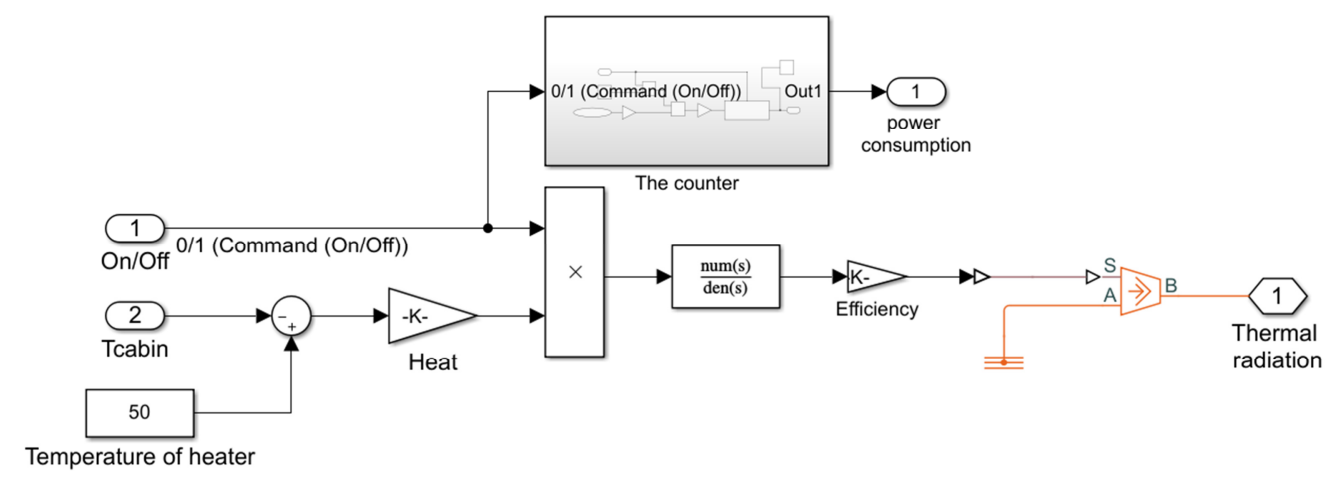

Fig.3. The model of the electric heater and the electricity counter

The heater consists of two branches: the upper one is a counter, the lower one is a heating element.

The operation of the counter is based on the calculation of the operating time of the electric heater.

The operation of the heater is based on the conversion of the difference between the temperature of the cabin and the temperature of the heater in thermal radiation. After that, the signal is sent from the comparison element to the signal amplifier. Then it is also multiplied with logical 1 or 0 . After the signal passes through the transfer function block "Transfer Fcn". Further, after the utilization coefficient, the signal is converted from a mathematical signal to a physical signal. After the physical signal arrives at the ideal temperature source. Here the final conversion of the physical signal into thermal signal occurs. The rating module is shown in Figure 4. 


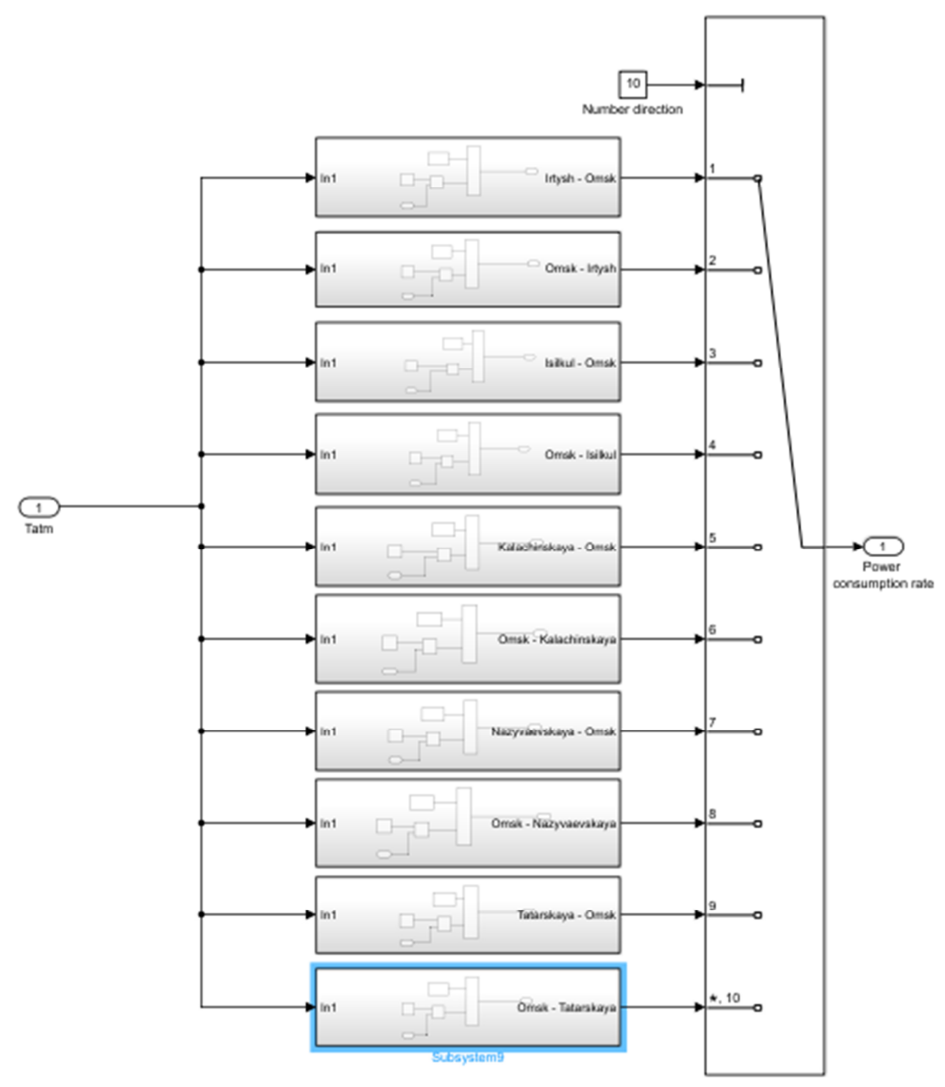

Fig.4. Power consumption rating module

The value of the ambient temperature comes to the input of the module. This signal arrives at one of ten subsystems. These subsystems are shown in Figure 5. At the output of the subsystem, the signal enters the "Multiport Switch" block, which is controlled through the "Position Number" block by the "Combo Box" block designed to control the rating module. The values and the method for determining the free coefficient and the coefficient of variable were presented in [7-10].

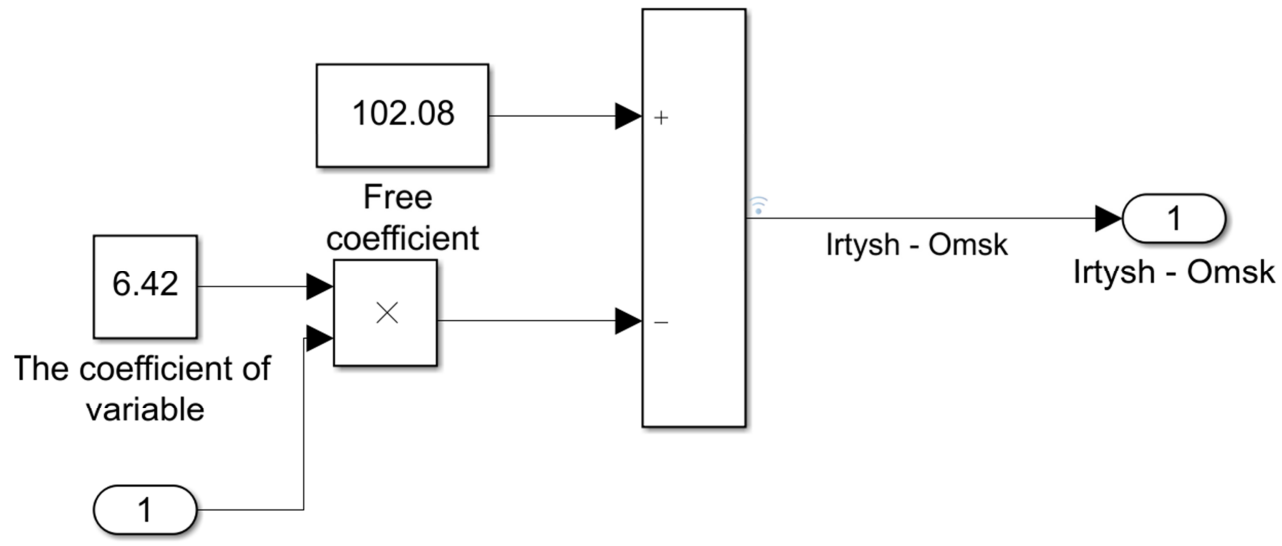

Fig. 5. Rating module subsystem based on statistical model 
At input 1, the value of the ambient temperature is fed. Then, passing through the multiplication unit, the signal from the temperature sensor is multiplied by the coefficient of variable t. The multiplied signal is fed to the adder, where the addition of the values of the free coefficient and the negative product occurs. As a result, a constant-time signal is obtained at the output, which shows the rate of electric power consumption for heating at an external temperature in a certain area. Figure 6 shows the model of the interior heating system.

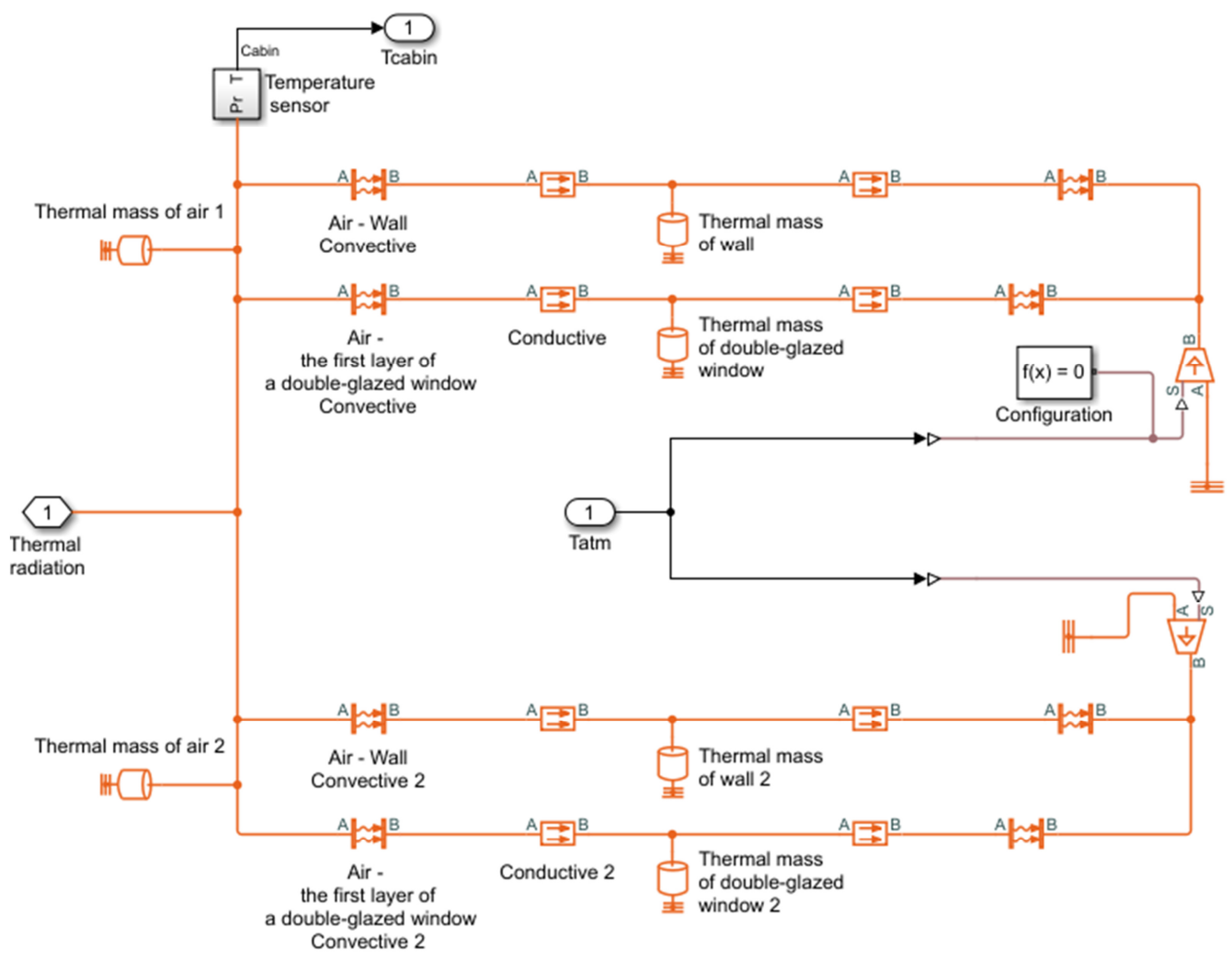

Fig.6. The model of the heating system of the ED4M electric train section

The model presented above is a physical model of the thermal processes that occur during heating of an electric train section. For conditions close to real, all the necessary data for the thermal model were calculated and found (thermal conductivity of materials, heat transfer, area of walls and windows, their thickness and others). Simplification has also been made, the walls are presented in the form of a single layer of insulation (URSA fiberglass).

During operation, most of the heat goes out through the windows and walls. Due to the fact that the thickness of the floor and roof of the car is greater than the thickness of windows and walls, it was decided to neglect them and exclude their loss from the model. Thus, there are only three components in the simulation model: air in the cabin, walls and windows.

The signal from the heater module is fed to thermal input 1 "Thermal radiation".

Thermal radiation acts primarily on air, which, through the convection process, transfers its heat to the first layer of glass and the first half of the wall thickness. Further, through heat transfer in solids, heat is transferred further to the second layer, which interacts with the environment and gives up all the stored energy. 
In the end, the signal is sent to a temperature sensor, in which it is converted from physical to mathematical (in the form of a temperature value in the interior).

After this signal goes to the thermostat, heater, and also enters the "Scope" unit. In the window of this block, the schedule of the system is obtained. The window of the "Scope" block is shown in Figure 7.

The upper graph shows the temperature of the interior, and the lower graph shows the ambient temperature.

It is seen that when the specified maximum or minimum temperature in the interior is reached, the thermostat reacts to this by turning the interior heating off or on, respectively.

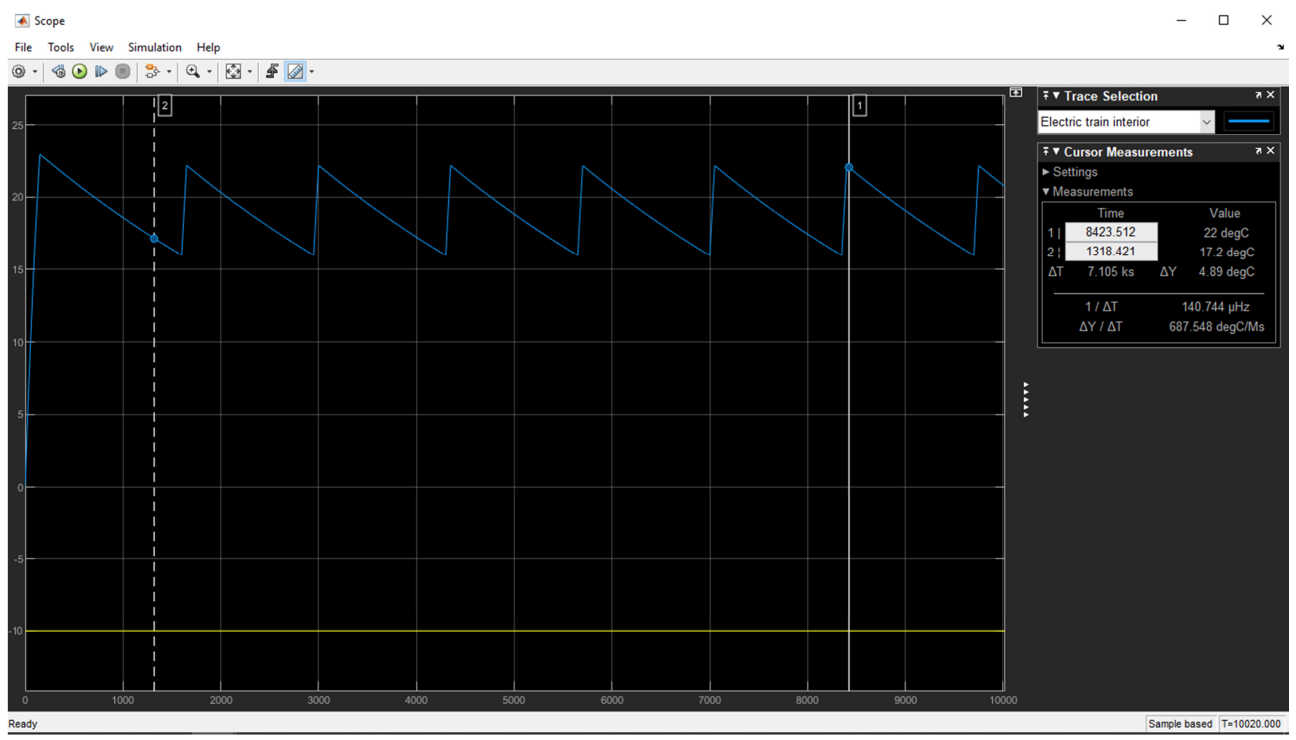

Fig.7. The window of the "Scope" block

\section{Conclusions}

In conclusion, it should be noted that the developed simulation model of a direct current electric train allows: selecting the parameters of the equipment of heating systems in terms of energy efficiency; controlling the energy consumption taking into account the factors that determine the energy-saving modes of its operation for the further establishment of the electric train sections with unsatisfactory technical condition; developing energy-efficient heating modes based on the analysis of data on energy consumption for heating of the whole train and of separate sections; identifying rational locations for temperature sensors; selecting thermal insulation materials for sections of electric trains.

As a perspective for developing the topic, it is proposed to improve the model in terms of taking into account the presence of passengers in the electric train interior, the consistency of other equipment of the electric train, taking into account the thermal conductivity of the roof and floor of the electric train, the processes of opening and closing doors, as well as the aerodynamic characteristics of cars, taking into account the influence of external factors such as snow and wind.

\section{References}

1. V. A. Gapanovich, Energy strategy and electrification of Russian railways (2012) 
2. D.A. Varfolomeev, V.I. Gorin, JSC Sci. Res. Des. Inst. Roll. St. Bull. No. 32 (2008)

3. V. A. Kuryatnikov, N. A. Polukhov, V. G. Glukhov, Appli. Pat. Ho. Bull., No. 14 (2013)

4. D.A. Gazev, E.M. Lytkina, A.M. Khudonogov, Bull., 22 (2013)

5. A.N. Lyutov, V.P. Moroz, A.V. Eremin, Appli. pat. hold. Bull, 22 (2009)

6. S.V. Kirilenko, M. A. Schneide, Appl. Pate. Hold. Bull., 15 (2012)

7. H. Lim, J. Jeong, Intern. J. Refr., 104, 229-245 (2019) doi:10.1504/IJHVS.2015.071689

8. S. Istomin, A. Perestenko, C. Dang, MATEC Web of Conferences, 239, (2018) https://doi.org/10.1051/matecconf/201823901035

9. S. Istomin, MATEC Web of Conferences, 239, (2018) https://doi.org/10.1051/matecconf/201823901038

10. S. G. Istomin, A. A. Straukhman, Trans. Ural., 4 (59), 81-85 (2018) DOI: 10.20291/1815-9400-2018-4-81-85. ISSN 1815-9400. 